\title{
Spectral Aspects of Bench-Scale Flammability Testing: Application to Hardwood Pyrolysis
}

\author{
MARCOS CHAOS \\ FM Global, Research Division \\ 1151 Boston-Providence Turnpike \\ Norwood, MA 02062, USA
}

\begin{abstract}
The anaerobic pyrolysis of wood material used to palletize commodities is studied in a Fire Propagation Apparatus (FPA) for a range of heating conditions relevant to fires. The data collected, consisting of mass loss rate, cumulative mass loss, and surface temperature, are used to determine model-specific material properties using inverse modeling and optimization methodologies previously developed in our laboratory. However, in this study, considerable effort is placed on determining the radiation environment that characterizes the FPA tests as well as how the radiation interacts with the samples. This is done on the basis of the recognition that boundary conditions have a pronounced effect on the output of a given pyrolysis model and, thus, the optimization results. The spectral radiance from the FPA heaters as well as the absorptivity/emissivity of the material surface are measured herein. The spectral features of the surface indicate that markedly different effective emissivities and absorptivities can be exhibited by the material depending on the spectral distribution of incident radiation. These effects are included in the pyrolysis model used to extract model-specific material properties so that the optimization process can, in a sense, be decoupled from boundary conditions. Therefore, it is expected that the approach described in this study can ensure that the derived model-specific properties can be applied to practical scenarios that are characterized by radiation environments that differ from those in bench-scale test apparatuses such as the FPA.
\end{abstract}

KEYWORDS: fire propagation apparatus, spectral radiation, emissivity, hardwood, pyrolysis, model, properties.

\section{INTRODUCTION}

Computational fluid dynamics (CFD) fire modeling tools have considerably advanced in recent years, e.g. $[1,2]$. These developments have allowed for the application of these tools to the study of fire phenomena at relatively large scales (e.g. [3, 4]), providing further understanding of the hazards associated with fires in industrial facilities. In these simulations, the rate of thermal degradation of the condensed-phase fuels involved in the fire is of great importance as it determines the heat release characteristics of the fire. Material "properties" are then needed that are suitable to the pyrolysis submodels, which provide the thermal degradation rate, used in the CFD simulations; the present work addresses this aspect.

In this study, the pyrolysis, in an inert environment, of wood pallet material is investigated in a Fire Propagation Apparatus (FPA). An approach similar to that outlined in [5] is followed in which modelspecific material properties are determined by inverse modeling and optimization applied to the pyrolysis dataset collected in the FPA. The methodology employed herein, however, differs from that of [5] in that careful attention is given to the spectral radiation environment of the tests by characterizing the radiative properties of the material as well as the FPA heating source. As shown in [6-8] for other opaque cellulosic materials, these properties determine the net absorbed heat flux received by the sample. It is noted that spectral characteristics of polymeric materials, although not the subject of the present study, and their interactions with various heating sources have also been studied (e.g. [9, 10]). Following this approach, the material properties obtained can be applicable to other practical scenarios that may be characterized by radiation sources that are spectrally different from those in the FPA. The data derived in this study will be useful in extending recent simulations [3,4], which considered idealized situations with unpalletized commodities, to be more representative of realistic warehouse storage scenarios. 


\section{EXPERIMENTAL}

\section{Fire Propagation Apparatus (FPA) pyrolysis experiments}

The Fire Propagation Apparatus used to collect bench-scale pyrolysis data is shown schematically in Fig. 1. The FPA used in the present experiments has been described in detail elsewhere (e.g. [5]) and only a brief description is provided here. The present apparatus is modeled after the device described in the ASTM 2058 and ISO 12136 standards [11, 12]. High-power high-density infrared tungsten halogen heaters (Research Inc., Hi-Temp 5209) are used which can provide heat fluxes approaching $120 \mathrm{~kW} / \mathrm{m}^{2}$ with uniformities of $8 \%$ over the sample surface. In addition, a humidity control and delivery system [13] is used to set the relative humidity (RH) of the gas supply to the FPA, which is maintained at $50 \pm 2.5 \%$ in the present experiments. Samples are placed in an insulated sample holder [14] and mounted horizontally on a load cell (0-1 kg range, $0.1 \mathrm{~g}$ accuracy, $0.02 \mathrm{~g}$ peak-to-peak noise). An infrared-grade quartz tube (1.65-cm ID, Quartz Scientific, Inc.) shields the sample and gasification products from room air entrainment. The gas supply, composed of $100 \% \mathrm{~N}_{2}$ in this study, is fed, using mass flow controllers, through a bed of glass beads to ensure flow uniformity. In the present experiments the flow provided to the system was 100 SLM.

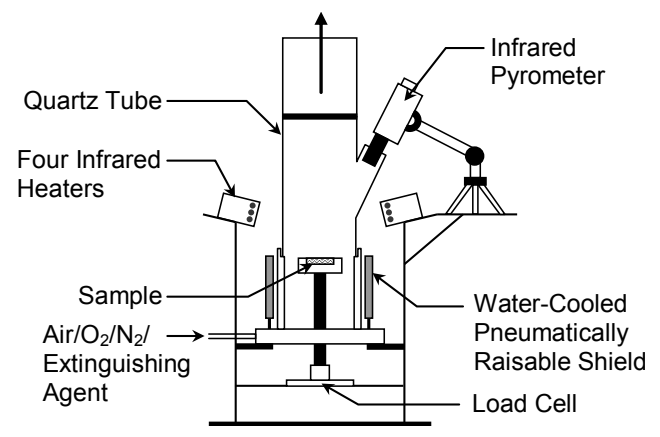

Fig. 1. The Fire Propagation Apparatus (FPA).

Surface temperatures are determined via an infrared pyrometer (Heitronics KT19.81-11, $223 \mathrm{~K}-1473 \mathrm{~K}$ range, $\sim 5 \mathrm{~K}$ accuracy). The pyrometer is sensitive in the $8-10 \mu \mathrm{m}$ range, which required modification of the quartz tube used (see Fig. 1) as quartz is not transparent at these wavelengths. However, use of the quartz tube (which transmits wavelengths up to approximately $5 \mu \mathrm{m}$, as shown below) ensures that radiation received by the pyrometer emanates from the sample and does not contain reflective components from sources external to the tube. For practical purposes, the emissivity setting of the pyrometer is set to 1 . Therefore, uncertainty remains as to the emissivity of the wood material used, both in its virgin and charred states. If the sample surface has an emissivity that deviates from unity, the pyrometer will underestimate the "true" surface temperature. The surface emissivity ranges between 0.8 and 1 in the $8-10 \mu \mathrm{m}$ range for both virgin material and char (see below). This implies that temperatures reported in the present study may underestimate the actual surface temperature by as much as $8 \%$ (see Ref. [15] for further details on the derivation of this value).

FPA experiments were performed by exposing conditioned samples (described below) to three heat flux levels; namely, 25, 50, and $100 \mathrm{~kW} / \mathrm{m}^{2}$. For each heat flux setting, three repeat experiments were conducted and data were averaged; test were repeatable to within $10 \%$. Data collected and considered herein consist of surface temperatures, mass loss, and mass loss rate (MLR). The latter were obtained by taking the derivative of the transient load cell mass loss data using Savitsky-Golay filters (e.g. [16-18]). The temporal size of the filter was kept between one and two times the full-width-half-magnitude size of the narrowest transient peak of interest [19] in order to avoid introduction of unwanted bias in the derived MLR data. This approach ensured that the magnitude of these peaks was preserved after application of the filter.

\section{Material}

The material tested in this study was wood obtained from standard 2-way pallets used in rack storage fire tests at FM Global's Research Campus facility in Rhode Island, USA. The type of wood used in the pallets was only vaguely specified as "mixed northern hardwoods" by the manufacturer. For the FPA experiments 
reported herein, circular samples $9.5 \mathrm{~cm}$ in diameter were used. Samples were first conditioned in a vacuum oven at $105^{\circ} \mathrm{C}$ for 24 hours to completely remove moisture. Subsequently, the samples were placed in the insulated sample holders and kept in an environmental chamber for 24 hours at $23^{\circ} \mathrm{C}$ and $50 \% \mathrm{RH}$. The resulting equilibrium moisture content of the samples was approximately $5 \%$ on a dry mass basis. It is noted that for the spectral characterization tests outlined in the following section, 2.54-cm diameter samples were used conditioned in the same manner as that described above. Furthermore, all samples were lightly sanded to ensure Lambertian surfaces.

\section{Spectral Characterization}

As discussed above, in this study attention was given to the nature of the radiation environment in the FPA tests as well as to how this radiation interacts with the sample surface. To this end, both the spectral radiance from the FPA radiant heating system and the spectral emissivity (absorptivity) of the wood pallet samples were measured.

The spectral radiance from the infrared heaters was measured in a line-of-sight manner by placing one of the heaters directly in front of a mid-infrared spectrometer [20] (Spectraline ES-200), See Fig. 2a. Incident radiation is dispersed within the spectrometer by a pair of calcium fluoride $\left(\mathrm{CaF}_{2}\right)$ prisms and imaged onto a 256-element lead selenide (PbSe) detector. With this arrangement, measurements in the 1-5 $\mu \mathrm{m}$ wavelength band were made with $20 \mathrm{~nm}$ resolution.

The spectral emissivity (absorptivity) of the wood pallet material was determined by measuring the surface reflectivity over a wide spectral range $(0.25-20 \mu \mathrm{m})$. This range is similar to that considered by Försth and Roos [7] and considerably broader than that used in the measurements of Wesson et al. [6] (0.3-5.5 $\mu \mathrm{m}$, approximately) and Boulet et al. [8] (1.4-10 $\mu \mathrm{m}$, approximately). A wide spectral range ensures that the fraction of blackbody (or greybody) emissive power contained within the spectral band is as high as possible for temperatures typical of fires and bench-scale tests, such as those conducted in the FPA. This fraction can be expressed as:

$$
F(\lambda, T)=\frac{2 \times 10^{24} \pi h_{p} c^{2}}{\sigma T^{4}} \int_{\lambda_{1}}^{\lambda_{2}}\left\{\lambda^{5}\left[\exp \left(\frac{1 \times 10^{6} h_{p} c}{\lambda k_{B} T}\right)-1\right]\right\}^{-1} d \lambda
$$

Where $F(\lambda, T)$ is the fraction of blackbody/greybody radiation in the $\lambda_{1} \leq \lambda \leq \lambda_{2}$ wavelength band at a given temperature $T(\mathrm{~K}), \lambda$ is the wavelength $(\mu \mathrm{m}), h_{p}$ is Planck's constant $\left(6.626 \times 10^{-34} \mathrm{~J} \mathrm{~s}\right), c$ is the speed of light $\left(2.998 \times 10^{8} \mathrm{~m} / \mathrm{s}\right), \sigma$ is the Stefan-Boltzmann constant $\left(5.6704 \times 10^{-8} \mathrm{~W} / \mathrm{m}^{2} / \mathrm{K}^{4}\right)$, and $k_{B}$ is Boltzmann's constant $\left(1.381 \times 10^{-23} \mathrm{~J} / \mathrm{K}\right)$. Figure 3 shows the results of Eq. 1 for temperatures between 500 and $3000 \mathrm{~K}$ and for the spectral ranges considered herein as well as by Wesson et al. [6] and Boulet et al. [8]. In evaluating the integral in Eq. 1 polylogarithmic functions were used, as described by Clark [21]. Note that, for all temperatures considered, in excess of $90 \%$ of the emissive power is contained within the chosen spectral band in this study as opposed to that within $0.3-5.5 \mu \mathrm{m}$ [6] or 1.4-10 $\mu \mathrm{m}$ [8]. These results indicate that the $0.25-20 \mu \mathrm{m}$ spectral range is sufficient to determine the radiative properties of the material for most practical temperatures.

Two instruments were used to cover the spectral range noted above: (1) a Shimadzu UV-3600 UV-Vis-NIR $(0.25-2.5 \mu \mathrm{m})$ double-beam spectrophotometer equipped with a $6-\mathrm{cm}$ diameter integrating sphere coated with barium sulfate $\left(\mathrm{BaSO}_{4}\right)$ and fitted with lead selenide and photomultiplier detectors; (2) a Nicolet iS10 FT-IR spectrometer coupled with a 7.6-cm diameter gold-coated integrating sphere (Pike Technologies IntegratIR $\left.{ }^{\mathrm{TM}}\right)$ fitted with a mercury cadmium telluride $(\mathrm{HgCdTe})$ detector $(2-20 \mu \mathrm{m})$. For UV-Vis-NIR measurements a substitution method was employed wherein the reflectivity of the samples was compared to that of a calibrated reference (see Fig. 2b); measured values were corrected for substitution error [22]. For measurements in the mid-infrared regime a flipper mirror could be switched to illuminate a calibrated reference or the sample so that no substitution was needed (the so-called Taylor method [23], see Fig. 2c). With known reflectivity, the emissivity (absorptivity) of the surface is determined by invoking Kirchhoff's law [24] (emissivity = absorptivity $=1$-reflectivity). This approach assumes a completely opaque surface over the spectral range considered, a fact that was verified in this study by measuring the transmissivity of 
the samples using the apparatuses described above. Transmissivity was negligible over the wavelength range studied.

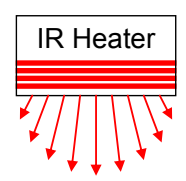

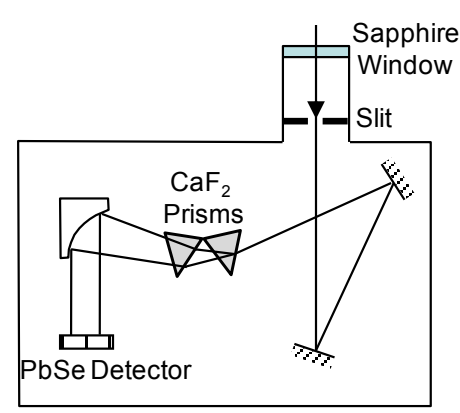

ES-200 Spectrometer

(a)

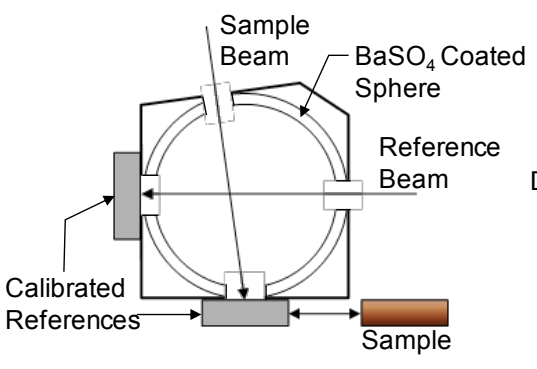

(b)

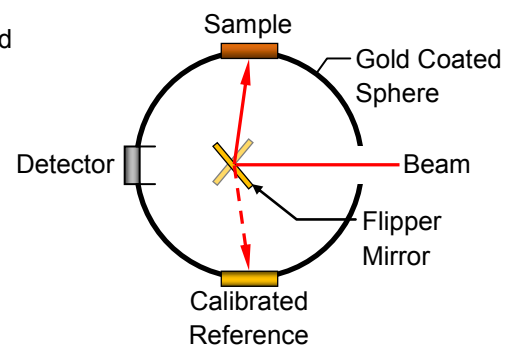

(c)

Fig. 2. Spectral measurements: (a) FPA radiant heater emission; (b) integrating sphere for sample reflectivity measurements in the $0.25-2.5 \mu \mathrm{m}$ spectral range; (c) integrating sphere for sample reflectivity measurements in the $2-20 \mu \mathrm{m}$ spectral range. The detectors for (b) are located $90^{\circ}$ out of plane from the sample and reference.

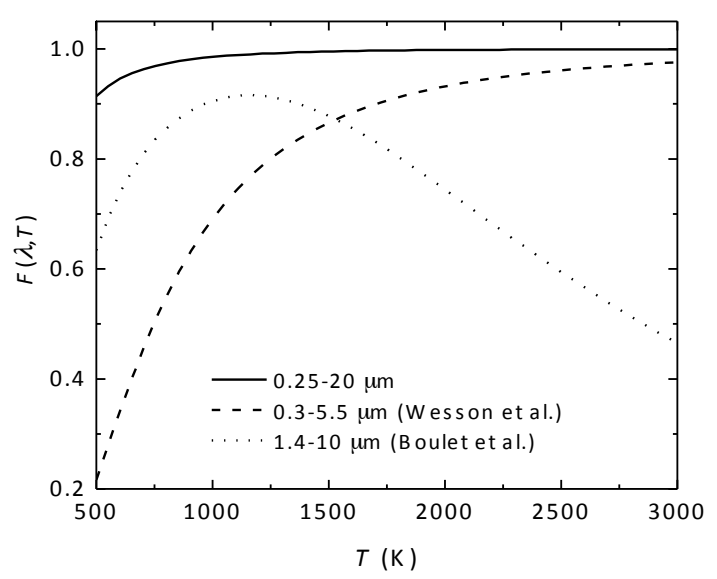

Fig. 3. Fraction of blackbody/greybody emissive power for different spectral ranges as a function of source temperature.

All reflectivity measurements were performed by illuminating the sample at $8^{\circ}$ from its surface normal and collecting the total hemispherical reflectivity. It is noted that no attempt was made to separate the measured reflectivity into diffuse and specular components; all surfaces are assumed to be Lambertian (i.e. diffuse) so that specularly reflected light is negligible compared to the total hemispherical value. Furthermore, measurements were performed at room temperature and it was assumed that the spectral reflectivity/emissivity/absorptivity of the material did not significantly change with surface temperature. As opposed to conducting or semi-conducting materials where electron movement (to fill conduction bands, for example) changes as a function of temperature, no such physical processes that may alter the surface characteristics occur in dielectric materials, such as the wood considered in this study. Therefore, the assumption of temperature independence is reasonable. Two sets of scans were taken for each measurement: one for the calibrated reference (i.e. the "background" reading) immediately followed by the sample scan. The calibrated reference used for the ultraviolet, visible, and near infrared measurements was a NIST traceable Labsphere Spectralon ${ }^{\circledR}$ certified diffuse reflectance standard (component SRS-99-010, serial \# 7A37B-4165); for the mid-infrared measurements a NPL (National Physics Laboratory, UK) 
traceable Avian gold certified diffuse reflectance standard (model RS-Au-02c, calibration \# AT-20121201IR1) was used. The uncertainty in the calibration values of the references is $2 \%$; the uncertainty in the spectral measurements reported herein is estimated to be $5 \%$ due to photometric accuracy of the detectors used.

\section{DISCUSSION OF RESULTS}

\section{Spectral characteristics of FPA heaters}

The measured spectral radiance from the FPA infrared heater is shown in Fig. 4a. Measurements were performed at various voltages applied to the heater corresponding to a specific heat flux setting (assuming a completely absorptive surface) determined in a separate calibration. Heat fluxes ranging from 10 to $100 \mathrm{~kW} / \mathrm{m}^{2}$ were considered. The FPA heater is, in essence, a series of tungsten filaments encased in quartz tubes filled with halogen gas. As a first approximation, it was assumed that the heater would behave as a greybody radiator the spectral radiance of which could reasonably be represented by Planck's equation:

$$
\varepsilon_{l} I(\lambda, T)=\varepsilon_{l} \frac{2 \times 10^{21} h_{p} c^{2}}{\lambda^{5}}\left[\exp \left(\frac{1 \times 10^{6} h_{p} c}{\lambda k_{B} T}\right)-1\right]^{-1}
$$

Where $I$ is the spectral radiance $\left(\mathrm{kW} / \mathrm{m}^{2} / \mu \mathrm{m} / \mathrm{sr}\right)$ and $\varepsilon_{l}$ is the emissivity of the heater lamp assembly (independent of wavelength for a grey body); all other quantities have been defined above. Equation 2 was fitted to the data shown in Fig. 4 a by treating $\varepsilon_{l}$ and $T$ (i.e. the heater lamp emissivity and effective emission temperature for each heat flux setting) as adjustable parameters. The fitted results are shown in Fig. 4a as lines along with the parameters obtained; reasonably good agreement can be observed. Note the relatively high color temperatures obtained for the largest heat fluxes considered (e.g. $T \approx 3000 \mathrm{~K}$ for $100 \mathrm{~kW} / \mathrm{m}^{2}$ ). The emissivity value determined for the heater $\left(\varepsilon_{l}=0.113\right)$ is consistent with reported emissivities for tungsten [25]. Further evidence of the near greybody behavior of the heater is provided by Fig. 4b. Considering the form of Eq. 2, dividing $\varepsilon_{l} I(\lambda, T)$ by $T^{5}$ yields a function which exclusively depends on $\lambda T$. Therefore, if the radiant intensities shown in Fig. 4a originated from a grey body all curves would collapse when normalized by $T^{5}$. Figure $4 \mathrm{~b}$ shows that, indeed, this is the case. Note that in Fig. $4 \mathrm{~b} \varepsilon_{l} I(\lambda, T)$ has been divided by $\sigma T^{5}$ for convenience, where $\sigma$ is the Stefan-Boltzmann constant. The variations shown at longer wavelengths may be related to wavelength dependencies of tungsten emissivity [25] as well as the interaction of radiation with the quartz surrounding the tungsten filament.
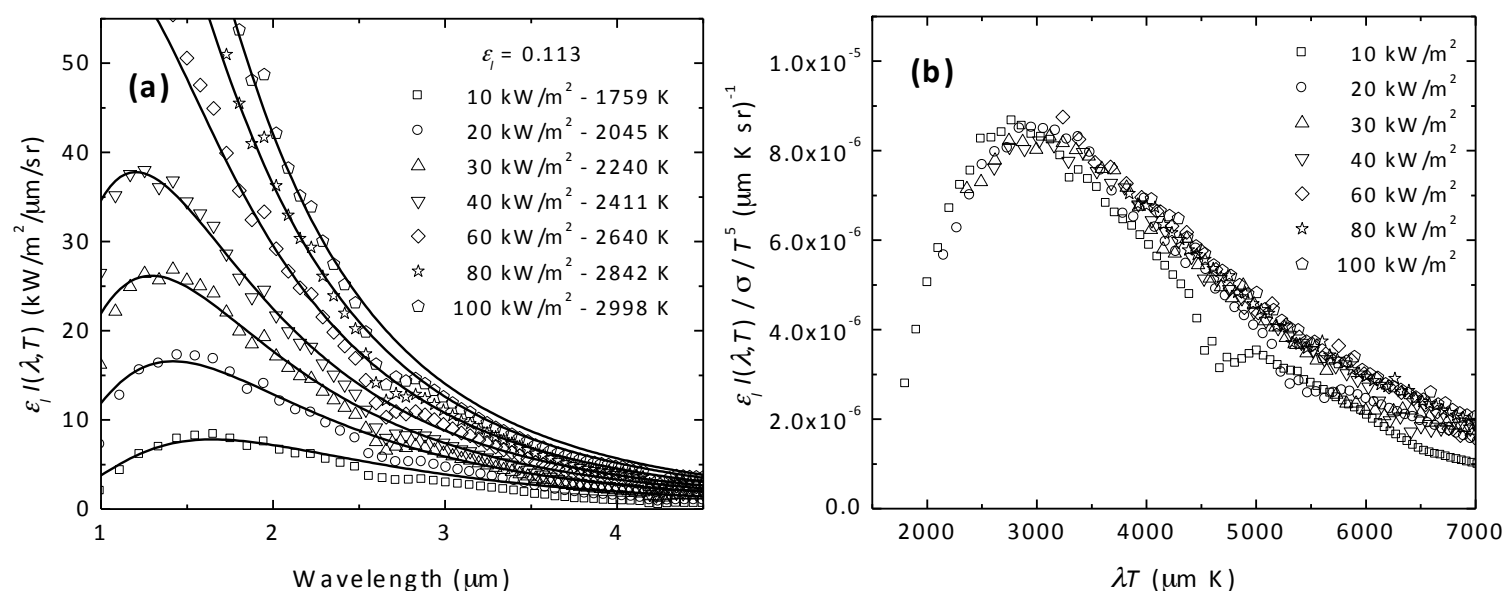

Fig. 4. (a) Spectral radiance measurements of FPA infrared heater at several heat flux settings (symbols) along with fitted results using Eq. 2 and the parameters listed in the legend; (b) plot of data in (a) normalized by $\sigma T^{5}$ as a function of the product of wavelength and temperature. 


\section{Emissivity/absorptivity of wood pallet material}

Spectral emissivity, $\varepsilon(\lambda)$, and absorptivity, $\alpha(\lambda)$, values (recall that $\varepsilon(\lambda)=\alpha(\lambda)$ ) determined from reflectivity data using the methodology described above are shown in Fig. 5 for the hardwood pallet material in both virgin and charred states. The charred sample was obtained by exposing a sample of virgin material to $50 \mathrm{~kW} / \mathrm{m}^{2}$ in the FPA (in a $\mathrm{N}_{2}$ environment) for approximately 10 minutes to completely char the surface and generate a relatively thick char layer. For comparison, data measured for Masonite by Wesson et al. [6] and for oak by Försth and Roos [7] are also plotted. For the virgin materials differences are observed in the visible and near infrared regions of the spectrum mainly due to color variations; however, beyond approximately $1.5 \mu \mathrm{m}$ emissivity is nearly identical for all materials. Results for plywood measured by Boulet et al. [8] also follow the trends shown in Fig. 5 and are not included for the sake of clarity. This is an interesting observation the reason for which is beyond the scope of the present study; however, it is likely due to the fact that these materials share similar chemical compositions (i.e. they are all lignocellulosic materials).

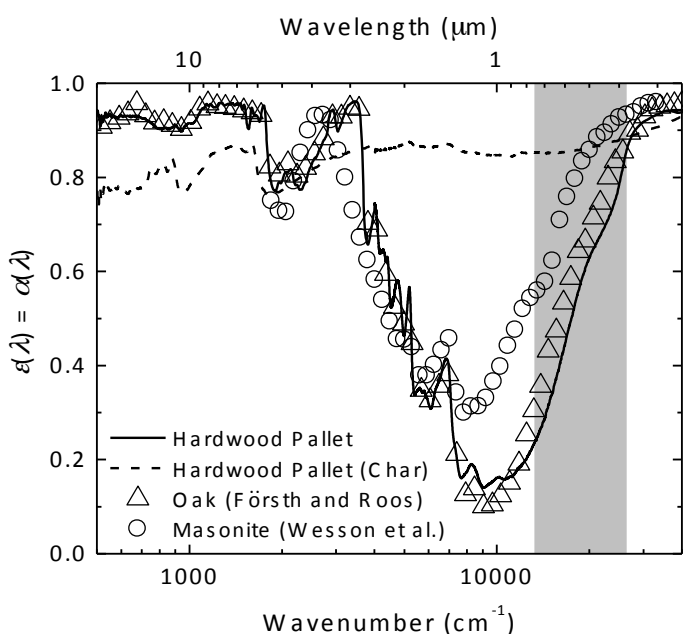

Fig. 5. Measured spectral emissivities of virgin (solid line) and charred (dashed line) pallet material compared to those for Masonite [6] and oak [7] (symbols). The visible wavelength regime is noted by the shaded region.

The data shown in Fig. 5 indicate that the wood pallet material is bound to absorb and emit radiation differently depending on the spectral distribution of the radiation interacting with it as well as the temperature of the material. For example, at wavelengths below $2 \mu \mathrm{m}$ (i.e. higher radiation temperatures) the spectral emissivity/absorptivity of the wood material may be as low as 0.2 . On the other hand, at longer wavelengths (lower radiation temperatures) the spectral emissivity/absorptivity increases to values above 0.8 and tends to remain spectrally flat. Therefore, one needs to determine the effective emissivity (absorptivity) of the wood in order to perform thermal radiation balances at the surface of the material.

A simplified schematic of the thermal radiation environment in the FPA is shown in Fig. 6a. The infrared lamps radiate at a specified temperature, $T_{l}$, depending on the specific heat flux setting (see Fig. 4). The spectral radiance of the lamps, $I\left(\lambda, T_{l}\right)$, interacts with the quartz tube surrounding the sample (see Fig. 1) so that the radiance falling on the sample surface is modified by the spectral transmissivity of quartz, $\tau(\lambda)$; it is this radiance that will be absorbed according to the spectral emissivity/absorptivity distribution of the material surface. On the other hand, thermal re-radiation from the sample will depend on the surface temperature, $T_{s}$. Therefore, the effective absorptivity, $\alpha_{e f f}\left(T_{l}\right)$, and effective emissivity, $\varepsilon_{e f f}\left(T_{s}\right)$, can be defined as:

$\alpha_{e f f}\left(T_{l}\right)=\frac{\int \alpha(\lambda) \tau(\lambda) I\left(\lambda, T_{l}\right) d \lambda}{\int \tau(\lambda) I\left(\lambda, T_{l}\right) d \lambda}$ 
$\varepsilon_{e f f}\left(T_{s}\right)=\frac{\int \varepsilon(\lambda) I\left(\lambda, T_{s}\right) d \lambda}{\int I\left(\lambda, T_{s}\right) d \lambda}$

Note that no temperature dependence has been assumed for $\varepsilon(\lambda)$ and $\alpha(\lambda)$, as argued above, as well as for transmissivity. Equations 3 and 4 simply express the average absorptivity and emissivity weighted by the black body spectrum at the specific temperature of interest. Note that in Eqs. 3 and 4, by Kirchhoff's law, $\varepsilon(\lambda)=\alpha(\lambda)$, as repeatedly mentioned above; however, $\varepsilon_{\text {eff }}$ may not necessarily be equal to $\alpha_{e f f}$; this is especially the case for materials that exhibit such spectral variations as those shown in Fig. 5 for the wood pallet material. Consider, for example, Fig. $6 \mathrm{~b}$ which shows the spectral emissivity/absorptivity of the wood material and the spectral transmissivity of the FPA quartz tube, which was measured using the devices described above, plotted against normalized spectral radiance at $T_{s}=500 \mathrm{~K}$ and $T_{l}=2500 \mathrm{~K}$. These temperatures approximately correspond, respectively, to the surface temperature at the onset of pyrolysis (as will be shown in the next section) and to a FPA lamp setting of $50 \mathrm{~kW} / \mathrm{m}^{2}$, see Fig. $4 \mathrm{a}$. Application of Eqs. 3 and 4 to the data shown in Fig. 6b yields $\alpha_{\text {eff }}\left(T_{l}\right)=0.35$ and $\varepsilon_{\text {eff }}\left(T_{s}\right)=0.90$.

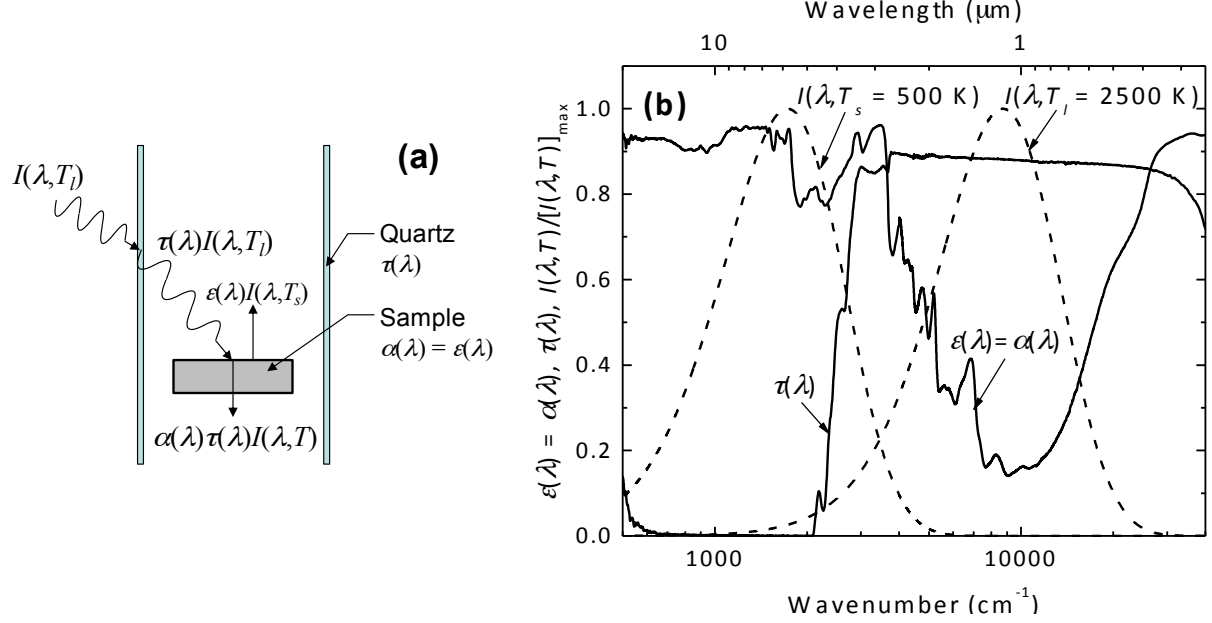

Fig. 6. (a) Thermal radiation in the FPA; (b) Normalized spectral radiance intensity curves (dashed lines) for representative temperatures compared to the emissivity/absorptivity of wood pallet material and the transmissivity of the FPA quartz tube (solid lines).

On the basis of the above discussion, Fig. 7 shows the results of Eqs. 3 and 4 as a function of temperature for the hardwood considered herein in its virgin and charred states. Note that the material exhibits markedly different effective absorptivity and emissivity values for the temperature ranges typically observed in a FPA test. The FPA heaters radiate at temperatures of $2000 \mathrm{~K}<T_{l}<3000 \mathrm{~K}$ which corresponds, on average, to an effective absorptivity of 0.40 . On the other hand, surface temperatures are characterized by $300 \mathrm{~K}<T_{s}<1200 \mathrm{~K}$ for which the effective emissivity is 0.85 , on average. For char, the effective absorptivity and emissivity are similar and remain fairly constant as a function of temperature, due to the relatively flat spectral profile shown in Fig. 5 for char. Note that at lower temperatures effective values for char are lower than those for the virgin material which is somewhat counterintuitive; this is due to the fact that lower temperatures are characterized by spectral radiance distributions which are biased towards longer wavelengths where the spectral emissivity of char is lower, see Fig. 5. These results will have direct implications in modeling FPA pyrolysis data particularly for defining a boundary condition for the net surface heat flux. Furthermore, the same implications would apply to fire environments where surface and flame temperatures may considerably differ.

\section{FPA pyrolysis data and optimized model parameters}

Pyrolysis data, consisting of mass loss rate (MLR) and surface temperature, collected in this study are shown in Fig. 8. As heat flux is increased the MLR curves become similar denoting a thermally thick material response. In general, all MLR curves in Fig. 8 show similar behavior, qualitatively. After an initial 
peak, the formation of an insulating char layer at the sample surface decreases the rate of gasification. Towards the end of the pyrolysis process, the mass loss rate increases before pyrolysis ceases, due to the presence of an insulating boundary. This latter behavior is apparatus dependent.

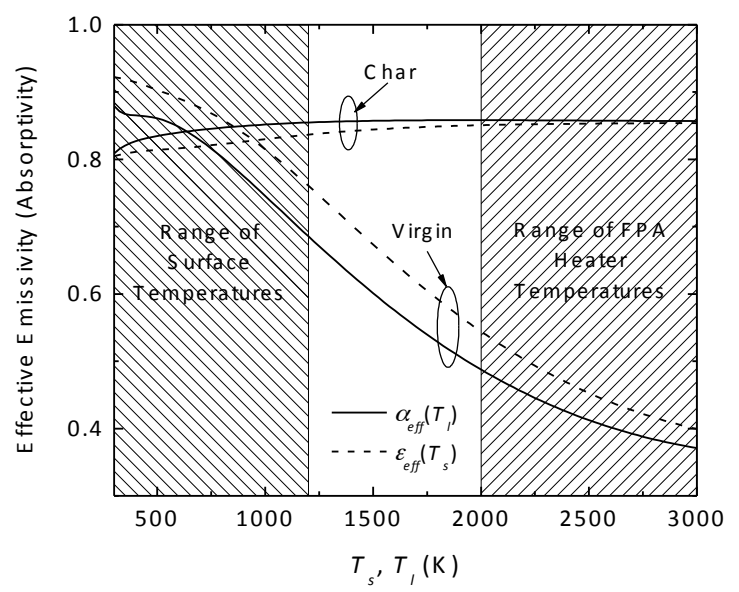

Fig. 7. Effective emissivity and absorptivity of the wood pallet material (virgin and char) as a function of temperature. Typical temperatures for surface emission and FPA radiation are shown by the shaded areas.
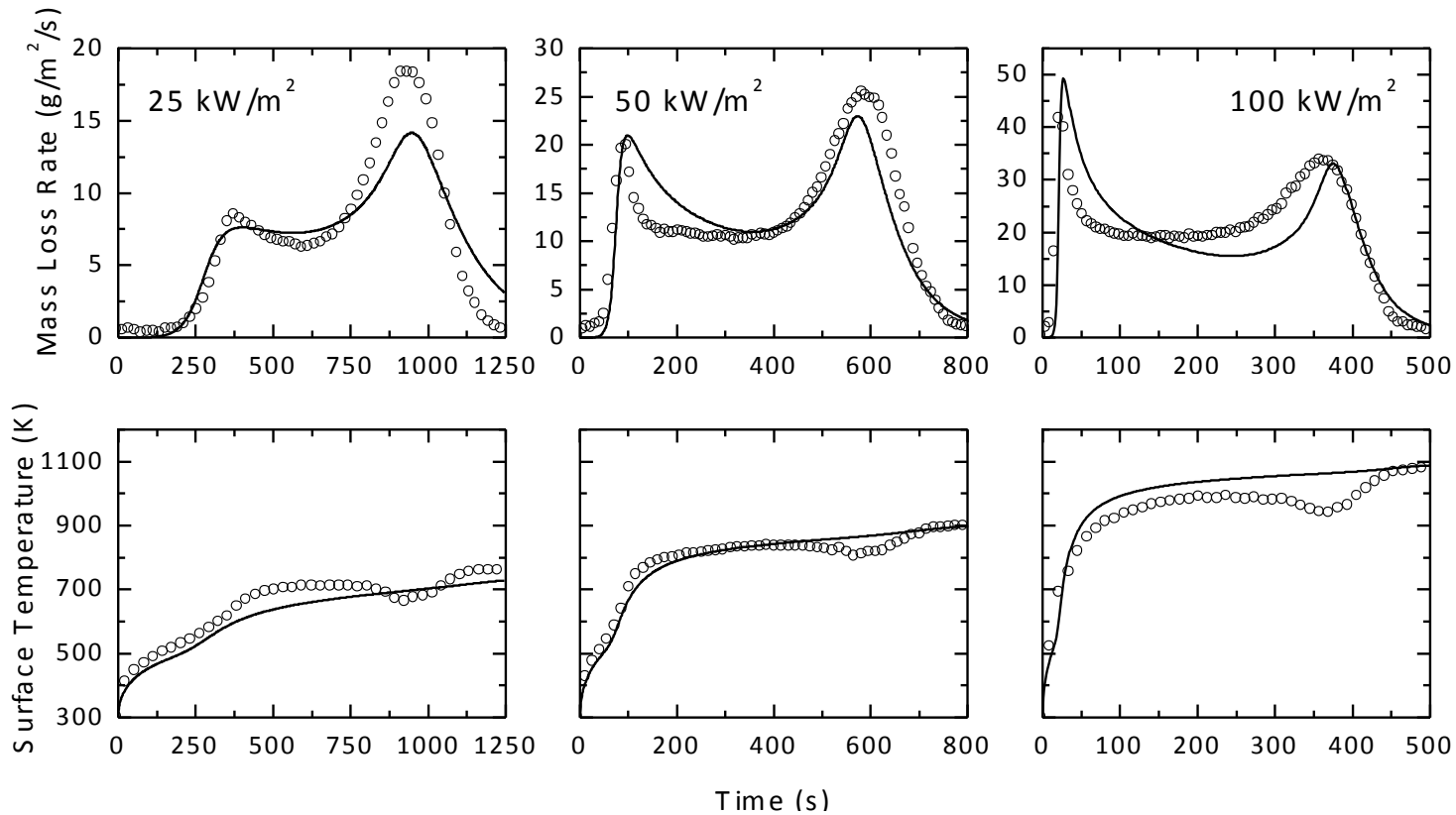

Fig. 8. Mass loss rate (top) and surface temperature (bottom) as a function of imposed external heat flux during pyrolysis of wood pallet material in an inert atmosphere. Symbols are experimental data; lines show pyrolysis model results using the optimized material properties listed in Table 1.

Surface temperatures shown in Fig. 8 exhibit a noticeable increase near 250, 70, and $20 \mathrm{~s}$ for 25, 50, and $100 \mathrm{~kW} / \mathrm{m}^{2}$, respectively. This is attributed to a change in the radiative properties of the sample surface and can be explained by considering the data shown in Figs. 5 and 7. For the range of FPA heater temperatures, virgin material and char have markedly different effective surface absorptivities. As the surface chars, the absorptivity increases and more radiant flux is absorbed, considerably raising the surface temperature. Conversely, one could argue that the behavior exhibited by the measured temperature may be due to an artifact possibly introduced by a change in surface emissivity that cannot be accounted for by the pyrometer, the emissivity setting of which is fixed at a constant value. Consider, however, that for the range of surface temperatures shown in Fig. 8 the effective emissivities are very similar for virgin material and 
char, see Fig. 7. Therefore, the radiant surface emission measured by the pyrometer is strictly a function of the surface temperature and the temperatures shown in Fig. 8 do indeed represent the change in absorptivity explained above. Finally, one further feature of the temperature data of Fig. 8 is that equilibrium temperatures reached by the surface are highly dependent on the imposed heat flux (i.e. $\sim 700, \sim 800, \sim 1000$ $\mathrm{K}$ for 20,50 , and $100 \mathrm{~kW} / \mathrm{m}^{2}$, respectively), as expected.

Similar to [5] optimization approaches are employed here to yield the model-specific or "effective" material properties that would be needed by a given pyrolysis model. These are obtained by adjusting the model input parameters (i.e. the material properties) in order to generate the best possible agreement between simulations and data shown in Fig. 8. It is expected that this approach can ensure that the outputs of the pyrolysis model (namely, MLR, cumulative mass loss, and surface temperature) when used in a CFD simulation at practical scales, are realistic over as wide a range of conditions as possible. In fact, a similar approach, applied to flame spread over corrugated cardboard panels [26], yielded satisfactory results. The Shuffled Complex Evolution (SCE) approach, an evolutionary optimization methodology [27], is used to recover the pyrolysis model parameters. This approach is selected due to its high accuracy, efficiency, and robustness [5, 28, 29]. Further details regarding the optimization technique can be found in Refs. 5 and 28.

The pyrolysis model used to describe the data of Fig. 8 is a simplified one-dimensional model, detailed in [5], based on the principles of [30]. A control volume approach is employed and the governing mass and energy conservation equations are solved numerically using a fully implicit scheme. Specific to the wood material under study herein the following general relationships apply:

$$
\begin{array}{ll}
\text { Virgin } \rightarrow \frac{\rho_{c}}{\rho_{v}} \text { Char }+\left(1-\frac{\rho_{c}}{\rho_{v}}\right) \text { Pyrolysate } & \text { solid reaction } \\
\frac{\partial}{\partial t}\left(\rho c_{p} T\right)=\frac{\partial}{\partial x}\left(k \frac{\partial T}{\partial x}\right)+\dot{\omega}_{v}^{\prime \prime \prime} \Delta H_{p, v} & \text { energy conservation } \\
\frac{\partial}{\partial t}\left(\rho Y_{v}\right)=\dot{\omega}_{v}^{\prime \prime \prime} & \text { mass conservation } \\
\dot{\omega}_{v}^{\prime \prime \prime}=\left[\frac{\rho Y_{v}}{\left(\rho Y_{v}\right)_{0}}\right]^{n}\left(\rho Y_{v}\right)_{0} A \exp \left(-\frac{E_{a}}{R T}\right) & \text { volumetric mass consumption rate } \\
M L R=\left(1-\frac{\rho_{c}}{\rho_{v}}\right)_{0}^{L} \dot{\omega}_{v}^{\prime \prime \prime} d x & \text { mass loss rate }
\end{array}
$$

Where $\rho_{v}$ and $\rho_{c}$ are, respectively, the densities $\left(\mathrm{kg} / \mathrm{m}^{3}\right)$ of virgin material and char. $Y_{v}$ is the mass fraction of virgin material; in each control volume the char mass fraction is given by $1-Y_{v}$. Density $(\rho)$ and thermal conductivity $(k, \mathrm{~W} / \mathrm{m} / \mathrm{K})$ are bulk values averaged by the volume fractions of virgin and char, while specific heat $\left(c_{p}\right)$ is averaged based on mass fractions. $\Delta H_{p, v}$ is the heat of pyrolysis $(\mathrm{J} / \mathrm{kg}), n$ is the reaction order, $A$ is the pre-exponential factor $\left(\mathrm{s}^{-1}\right), R$ is the universal gas constant $(8.314 \mathrm{~J} / \mathrm{mol} / \mathrm{K}), E_{a}$ is the activation energy $(\mathrm{J} / \mathrm{mol})$, and $T$ is the temperature $(\mathrm{K})$. The subscript 0 designates initial conditions before heating and pyrolysis. $L$ is the thickness of the solid (m) and $t$ and $x$ are temporal and spatial variables. All physical properties are assumed to be temperature independent. The pyrolysate is assumed to escape the solid immediately upon formation with no heat exchange with the solid. Equations 5-9 describe the singlestep Arrhenius charring process of a homogeneous material where only three species are treated: virgin solid, char, and pyrolysate.

The pyrolysis scheme above is subject to boundary conditions that describe heat balances at both the front and back surfaces of the sample, see Fig. 9. As described above the samples used in the FPA tests were insulated; the heat loss to the insulation, $\dot{q}_{\text {loss }}^{\prime \prime}$, has been previously described numerically by de Ris and Khan [14] and this treatment is included in the present pyrolysis model. For the front surface, however, the heat balance is described differently in this study compared to Refs. 5 and 28 , which defined the net heat flux at the surface as: 
$\dot{q}_{n e t}^{\prime \prime}=\bar{\varepsilon}\left\lfloor\dot{q}_{e x t}^{\prime \prime}-\sigma\left(T_{s}^{4}-T_{\infty}^{4}\right)\right\rfloor-h\left(T_{s}-T_{\infty}\right)$

Where $\varepsilon,{ }^{-}$is the surface emissivity, which is an average between virgin and char emissivities weighted by their respective volume fractions present at the surface; $\dot{q}_{e x t}^{\prime \prime}$ is the externally imposed heat flux from the FPA heaters, $T_{s}$ and $T_{\infty}$ are, respectively, surface and ambient temperatures; and $h$ is a convection coefficient. Previous optimization efforts $[5,28]$ considered virgin and char emissivities to be part of the optimized property parameter set. Furthermore, Eq. 10 assumes that the surface is grey so that the effective absorptivity and emissivity are equal. However, it has been shown above that this is hardly the case for the wood pallet material of interest in this study; therefore, following Fig. 9, the pyrolysis model has been modified to express the surface heat balance as follows:

$\dot{q}_{n e t}^{\prime \prime}=\bar{\alpha}_{e f f}\left(T_{l}\right) \dot{q}_{e x t}^{\prime \prime}-\bar{\varepsilon}_{e f f}\left(T_{s}\right) \sigma\left(T_{s}^{4}-T_{\infty}^{4}\right)-h\left(T_{s}-T_{\infty}\right)$

Where all variables have been previously defined; $\alpha$, ${ }_{\text {eff }}^{-}\left(T_{l}\right)$ and $\varepsilon$, ${ }_{\text {eff }}^{-}\left(T_{s}\right)$ denote the average effective absorptivity and emissivity (Eqs. 3 and 4), respectively, weighted by the volume fractions of virgin material and char, as mentioned above. Given the data shown in Fig. 7, it was assumed that $\alpha_{e f f}\left(T_{l}\right)=\varepsilon_{e f f}\left(T_{s}\right)=0.85$ for char, regardless of temperature. By introducing these changes into the pyrolysis model, the surface heat balance boundary condition is fully described. This is done in an attempt to decouple the material properties obtained by optimization from the experimental conditions specific to the FPA. Furthermore, it is noted that the phenomena described above and taken into account in developing Eq. 11 should be considered when interpreting data from other bench-scale testing venues, such as the cone calorimeter.

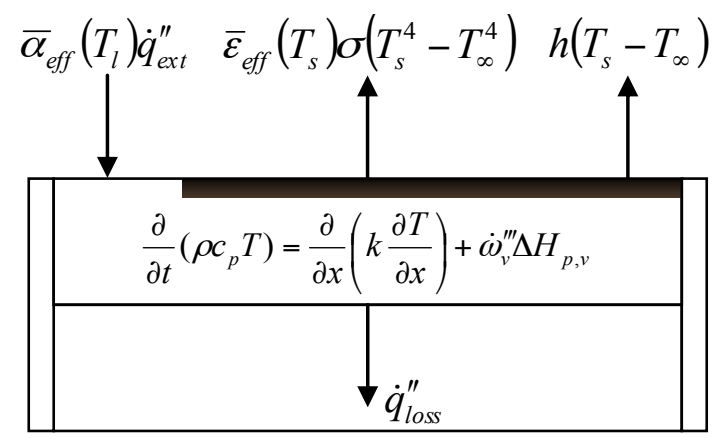

Fig. 9. One-dimensional heat balance for a wood pallet sample in an insulated holder.

The material properties obtained following the methodology described above are listed in Table 1 . The density of the virgin material was determined from weight and size measurements and was not optimized. Furthermore, separate sensitivity analyses [31] showed that the heat capacity of char is not a parameter to which MLR, cumulative mass loss, and surface temperature are sensitive under the assumptions and conditions of the present study. Therefore, in order to reduce the dimensionality of the optimization problem, the char heat capacity was set to $1450 \mathrm{~J} / \mathrm{kg} / \mathrm{K}$ which is the heat capacity of carbon (graphite) [32] evaluated at an average value for the surface temperatures shown in Fig. 8.

The values obtained for the physical properties shown in Table 1, although being "effective" and model dependent, are quite reasonable and agree well with published values for hardwoods [33]. For example, the room temperature thermal conductivity of several virgin hardwoods is reported to be within the range of $0.10-0.21 \mathrm{~W} / \mathrm{m} / \mathrm{K}$ [33]. The heat capacity for virgin material, on the other hand, varies between approximately $1250 \mathrm{~J} / \mathrm{kg} / \mathrm{K}$ at room temperature to $2500 \mathrm{~J} / \mathrm{kg} / \mathrm{K}$ at $600 \mathrm{~K}$ [33] (the approximate temperature, from Fig. 8 at which transition to char occurs). These properties are well approximated considering that the pyrolysis model assumes temperature-independent properties. Higher values are obtained by the SCE optimization algorithm, possibly to capture the presence of water in the sample [34]. It is noted that the value found for the heat of pyrolysis is slightly endothermic and nearly negligible. An overall thermally neutral pyrolysis reaction makes physical sense, given the simplifications made in the model, since wood pyrolysis consists of both endothermic and exothermic decomposition processes [35- 
38]. Furthermore, the kinetic reaction parameters shown in Table 1 are in good agreement with reported values for some woods [38].

Table 1. Optimized properties

\begin{tabular}{|c|c|c|}
\hline & Property & Value \\
\hline \multirow[t]{4}{*}{ Virgin } & Thermal Conductivity $\left(\mathrm{W} / \mathrm{m}^{2} / \mathrm{K}\right)$ & 0.26 \\
\hline & Density $\left(\mathrm{kg} / \mathrm{m}^{3}\right)$ & $660^{\mathrm{a}}$ \\
\hline & Heat Capacity $(\mathrm{J} / \mathrm{kg} / \mathrm{K})$ & 2533 \\
\hline & Emissivity/Absorptivity & Varies $^{\mathrm{b}}$ \\
\hline \multirow[t]{4}{*}{ Char } & Thermal Conductivity $\left(\mathrm{W} / \mathrm{m}^{2} / \mathrm{K}\right)$ & 0.36 \\
\hline & Density $\left(\mathrm{kg} / \mathrm{m}^{3}\right)$ & 100 \\
\hline & Heat Capacity $(\mathrm{J} / \mathrm{kg} / \mathrm{K})$ & $1450^{\mathrm{c}}$ \\
\hline & Emissivity/Absorptivity & $0.85^{\mathrm{d}}$ \\
\hline \multirow[t]{4}{*}{ Reaction } & Pre-exponential Factor $\left(\mathrm{s}^{-1}\right)$ & $5.13 \times 10^{14}$ \\
\hline & Activation Energy $(\mathrm{J} / \mathrm{mol})$ & $1.63 \times 10^{5}$ \\
\hline & Heat of Pyrolysis $(\mathrm{J} / \mathrm{kg})$ & -6.8 \\
\hline & Reaction Order & 6.02 \\
\hline \multicolumn{3}{|c|}{ Directly measured. } \\
\hline \\
\hline \multicolumn{3}{|c|}{$\begin{array}{l}\text { Derived from spectral measurements (see text). } \\
\text { Heat capacity of graphite (carbon) at the average surface temperature }\end{array}$} \\
\hline
\end{tabular}

The pyrolysis model results for MLR and surface temperature, using the properties in Table 1, are plotted against FPA data in Fig. 8; quite good agreement is observed. Determination coefficients (i.e. $\mathrm{R}^{2}$ values), averaged over the three heating conditions considered, are 0.85 for MLR, 0.99 for cumulative mass loss, and 0.83 for surface temperature. At lower heat fluxes and longer times the agreement degrades somewhat. This may be due to the fact that in the experiments at low heat fluxes, especially at $25 \mathrm{~kW} / \mathrm{m}^{2}$, the sample had a tendency to warp possibly losing contact with the sample holder. In addition, at low heat fluxes the effects of the presence of water (not modeled) are more prevalent.

In order to further evaluate whether the procedure described here can indeed properly decouple the optimization process from the boundary conditions, a second set of pyrolysis data was collected wherein the surface of the samples was coated with a paint of known emissivity/absorptivity (Thurmalox 250, Dampney Co. Inc.). The paint exhibits greybody behavior with a spectrally flat emissivity over a wide wavelength range and a value of approximately 0.92 . The mass loss rate data of the painted samples are shown in Fig. 10 compared to the model results of Fig. 8; temperatures are not shown for the sake of brevity. Since the paint significantly increases the initial absorptivity of the surface when exposed to the FPA heaters (from approximately 0.4 for a bare surface, see Fig. 7 , to 0.92 ) the onset of pyrolysis occurs at considerably earlier times, especially at low heat flux levels. Notably, however, is the fact that the pyrolysis model is able to capture the pyrolysis trends of the painted samples using the properties listed in Table 1 and setting the emissivities/absorptivities of virgin material and char to 0.92 . These results provide two indications: (1) the optimized properties can be used in scenarios characterized by considerably different boundary conditions; (2) results from the pyrolysis model using the optimized properties may be valid for effective heat flux levels beyond those used to obtain the properties. This latter conclusion stems from the fact that the heat flux initially absorbed by the surface is considerably different for the tests of Figs. 8 and 10. For example, for the highest heat flux setting $\left(100 \mathrm{~kW} / \mathrm{m}^{2}\right)$ the net heat flux absorbed by the bare surface (Eq. 11) at the initial pyrolysis stages is approximately $40 \mathrm{~kW} / \mathrm{m}^{2}$ whereas this value is approximately $90 \mathrm{~kW} / \mathrm{m}^{2}$ for the painted surface. The preceding discussion provides further support for the validity and practical use of the approach described herein.

\section{CONCLUSION}

In this work the anaerobic pyrolysis of wood used to palletize commodities has been studied in a Fire Propagation Apparatus (FPA) under a wide range of heat fluxes representative of practical fire scenarios. The data collected have been used to determine model-specific material properties through inverse 
pyrolysis modeling coupled to optimization techniques. These properties can be employed in future CFD modeling of flame spread in palletized commodities. Considerable effort was placed on the decoupling of the pyrolysis model and, thus, the obtained model-specific parameters from the physical boundary conditions. To this end, the spectral radiation environment of the tests was fully characterized by measuring the spectral radiance from the FPA heaters as well as the absorptivity/emissivity of the material surface. Rich spectral features have been shown for the wood pallet material, which alter the way the material effectively absorbs and emits radiation. The effect of this behavior on the net heat flux absorbed by the surface was included in the pyrolysis model. The updated pyrolysis model, along with the obtained optimized parameters, has been shown to not only reproduce the FPA data used for parameter optimization but also the pyrolysis behavior of samples with considerably different boundary conditions. The approach described herein ensures that the model-specific material properties derived can be applied to practical scenarios that are characterized by spectral radiation environments that differ from those in bench-scale flammability testing equipment, such as the FPA.
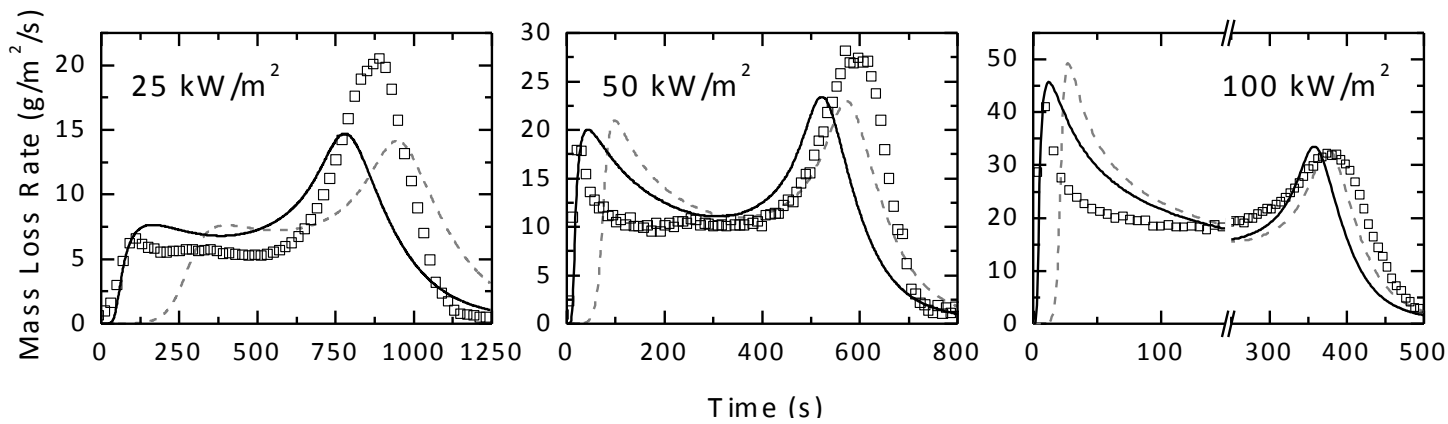

Fig. 10. Measured MLR for painted wood pallet material samples (symbols) as a function of imposed external heat flux. Black lines show pyrolysis model results using the properties listed in Table 1 along with emissivity/absorptivity values corresponding to the paint used. For comparison, model results corresponding to the conditions of Fig. 8 are shown as grey dashed lines.

\section{ACKNOWLEDGEMENTS}

This work was funded by FM Global and performed within the framework of the FM Global Strategic Research Program on Fire Modeling. The author is grateful to Mr. Lawney Crudup and Mr. Stephen Ogden for assistance in sample preparation and in FPA experiments, Dr. GearyYee for assistance in FT-IR measurements, and Dr. Sergey Dorofeev for helpful discussions.

\section{REFERENCES}

[1] Wang, Y., Chatterjee, P., and de Ris, J.L., (2011) Large Eddy Simulation of Fire Plumes, Proceedings of the Combustion Institute 33: 2473-2480, http://dx.doi.org/10.1016/j.proci.2010.07.031 (also see fmglobal.com/modeling)

[2] McGrattan, K., Hostikka, S., Floyd, J., Baum, H., Rehm, R., Mell, W., and McDermott, R., "Fire Dynamics Simulator (Version 5)," National Institute of Standards and Technology Special Publication 1018-5, Technical Reference Guide, 2008.

[3] Meredith, K.V., Chatterjee, P., Wang, Y., and Xin, Y., "Simulating Sprinkler Based Rack Storage Fire Suppression Under Uniform Water Application," Proceedings of the $7^{\text {th }}$ International Seminar on Fire and Explosion Hazards (ISFEH7), 2013, pp. 511-520. http://dx.doi.org/10.3850/978-981-07-5936-0_07-08

[4] Chatterjee, P., Wang, Y., Chaos, M., Zhou, X., Meredith, K.V., and Dorofeev, S.B., "Numerical Simulation of Fire Growth on Corrugated Cardboard Commodities in Three-Tier-High Rack Storage Arrays," Interflam 2013, June 24-26, 2013, Nr Windsor, UK.

[5] Chaos, M., Khan, M.M., Krishnamoorthy, N., de Ris, J.L., and Dorofeev, S.B., (2011) Evaluation of Optimization Schemes and Determination of Solid Fuel Properties for CFD Fire Models Using 
Bench-Scale Pyrolysis Tests, Proceedings of the Combustion Institute 33: 2599-2606, http://dx.doi.org/10.1016/j.proci.2010.07.018

[6] Wesson, H.R., Welker, J.R., and Sliepcevich, C.M., (1971) The Piloted Ignition of Wood by Thermal Radiation, Combustion and Flame 16: 303-310, http://dx.doi.org/10.1016/S00102180(71)80101-3

[7] Försth, M. and Roos, A., (2011) Absorptivity and its Dependence on Heat Source Temperature and Degree of Thermal Breakdown, Fire and Materials 35: 285-301, http://dx.doi.org/10.1002/fam.1053

[8] Boulet, P., Parent, G., Acem, Z., Rogaume, T., Fateh, T., Zaida, J., and Richard, F., (2012) Characterization of the Radiative Exchanges When Using a Cone Calorimeter for the Study of The Plywood Pyrolysis, Fire Safety Journal 51: 53-60, http://dx.doi.org/10.1016/j.firesaf.2012.03.003

[9] Hallman, J.R., Welker, J.R., and Sliepcevich, C.M., (1974) Polymer Surface ReflectanceAbsorbance Characteristics, Polymer Engineering and Science 14: 717-723, http://dx.doi.org/10.1002/pen.760141010

[10] Bal, N., Raynard, J., Rein, G., Torero, J.L., Försth, M., Boulet, P., Parent, G., Acem, Z., and Linteris, G., (2013) Experimental Study of Radiative Heat Transfer in a Translucent Fuel Sample Exposed to Different Spectral Sources, International Journal of Heat and Mass Transfer 61: 742748, http://dx.doi.org/10.1016/j.ijheatmasstransfer.2013.02.017

[11] ASTM E 2058-13, Standard Test Methods for Measurement of Synthetic Polymer Material Flammability Using a Fire Propagation Apparatus (FPA). ASTM International, West Conshohocken, PA, 2013, DOI: 10.1520/E2058-13, www.astm.org

[12] ISO 12136:2011, Reaction to Fire tests - Measurement of Material Properties Using a Fire Propagation Apparatus. International Organization for Standardization, Geneva, Switzerland.

[13] Khan, M.M., de Ris, J.L., and Ogden, S.D., (2009) Effect of Moisture on Ignition Time of Cellulosic Materials, Fire Safety Science 9: 167-178, http://dx.doi.org/10.3801/IAFSS.FSS.9-167

[14] de Ris, J.L. and Khan, M.M., (2000) A Sample Holder for Determining Material Properties, Fire and Materials 24: 219-226, http://dx.doi.org/10.1002/1099-1018(200009/10)24:5<219::AIDFAM741>3.0.CO;2-7

[15] Chaos, M., Khan, M.M., and Dorofeev, S.B., (2013) Pyrolysis of Corrugated Cardboard in Inert and Oxidative Environments, Proceedings of the Combustion Institute 34: 2583-2590, http://dx.doi.org/10.1016/j.proci.2012.06.031

[16] Savitzky, A. and Golay, M.J.E., (1964) Smoothing and Differentiation of Data by Simplified Least Squares Procedures, Analytical Chemistry 36: 1627-1639, http://dx.doi.org/10.1021/ac60214a047

[17] Khan, M.M. and de Ris, J.L., (2005) Operator Independent Ignition Measurements, Fire Safety Science 8: 163-174, http://dx.doi.org/10.3801/IAFSS.FSS.8-163

[18] Staggs, J.E.J., (2005) Savitzky-Golay Smoothing and Numerical Differentiation of Cone Calorimeter Mass Data, Fire Safety Journal 40: 493-505, http://dx.doi.org/10.1016/j.firesaf.2005.05.002

[19] Bromba, M.U.A. and Ziegler, H., (1981) Application Hints for Savitzky-Golay Digital Smoothing Filters, Analytical Chemistry 53: 1583-1586, http://dx.doi.org/10.1021/ac00234a011

[20] Ji, J., Gore, J.P., Sivathanu, Y.R., and Lim, J.M., (2004) Fast Infrared Array Spectrometer with a Thermoelectrically Cooled 160-Element PbSe Detector, Review of Scientific Instruments 75: 333 339, http://dx.doi.org/10.1063/1.1634362

[21] Clark, B.A., (1987) Computing Multigroup Radiation Integrals Using Polylogarithm-Based Methods, Journal of Computational Physics 70: 311-329, http://dx.doi.org/10.1016/00219991(87)90185-9 
[22] Jacquez, J.A. and Kuppenheim, H.F., (1955) Theory of the Integrating Sphere, Journal of the Optical Society of America 45: 460-470, http://dx.doi.org/10.1364/JOSA.45.000460

[23] Taylor, A.H., (1920) The Measurement of Diffuse Reflection Factors and a New Absolute Reflectometer, Journal of the Optical Society of America 4: 9, http://dx.doi.org/10.1364/JOSA.4.000009

[24] Kirchhoff, G., (1860) Über das Verhältnis zwischen dem Emissionsvermögen und dem Absorptionsvermögen. der Körper für Wärme und Licht, Poggendorfs Annalen der Physik und Chemie 109: 275-301. (English translation by Guthrie, F.: Kirchhoff, G., (1860) On the Relation between the Radiating and the Absorbing Powers of Different Bodies for Light and Heat, Philosophical Magazine Series 4 20: 1-21, http://dx.doi.org/10.1080/14786446008642901)

[25] Touloukian, Y.S. and DeWitt, D.P., Thermal Radiative Properties: Metallic Elements and Alloys, IFI/Plenum, New York, 1970.

[26] Chaos, M., Wang, Y., and Dorofeev, S.B., "CFD Modeling of Flame Spread over Corrugated Cardboard Panels," Proceedings of the International Congress on Fire Computer Modeling, October 18-19, 2012, Santander, Spain, pp. 15-33.

[27] Duan, Q.Y., Gupta, V.K., and Sorooshian, S., (1993) Shuffled Complex Evolution Approach for Effective and Efficient Global Minimization, Journal of Optimization Theory and Applications 76: 501-521, http://dx.doi.org/10.1007/BF00939380

[28] Chaos, M., Khan, M.M., Krishnamoorthy, N., de Ris, J.L., and Dorofeev, S.B., "FPA Bench-Scale Flammability Tests and Extraction of Solid Fuel Properties for Fire Models." Proceedings of the $6^{\text {th }}$ International Seminar on Fire and Explosion Hazards (ISFEH6), 2010, pp. 995-1007. http://dx.doi.org/10.3850/978-981-08-7724-8_15-01

[29] Lautenberger, C. and Fernández-Pello, A.C. (2011) Optimization Algorithms for Material Pyrolysis Property Estimation, Fire Safety Science 10: 751-764, http://dx.doi.org/10.3801/IAFSS.FSS.10-751

[30] Lautenberger, C. and Fernández-Pello, C., (2009) Generalized Pyrolysis Model for Combustible Solids, Fire Safety Journal 44: 819-839, http://dx.doi.org/10.1016/j.firesaf.2009.03.011

[31] Chaos, M., (2013) Application of Sensitivity Analyses to Condensed-Phase Pyrolysis Modeling, Fire Safety Journal 61: 254-264, http://dx.doi.org/10.1016/j.firesaf.2013.09.016

[32] Frenkel, M., Chirico, R.D., Diky, V., Muzny, C.D., Kazakov, A.F., and Lemmon, E.W. "ThermoData Engine Version 2.1 - Pure Compounds and Equations of State." NIST Standard Reference Database Number 103a, 2008.

[33] Glass, S.V. and Zelinka, S.L., "Moisture Relations and Physical Properties of Wood." Chapter 4 in Wood Handbook - Wood as an Engineering Material, Forest Products Laboratory General Technical Report FPL-GTR-190, 2010.

[34] Simms, D.L. and Law, M., (1967) The Ignition of Wet and Dry Wood by Radiation, Combustion and Flame 11: 377-388, http://dx.doi.org/10.1016/0010-2180(67)90058-2

[35] Roberts, A.F., (1971) The Heat of Reaction During the Pyrolysis of Wood, Combustion and Flame 17: 79-86, http://dx.doi.org/10.1016/S0010-2180(71)80141-4

[36] Di Blasi, C. (1993), Modeling and Simulation of Combustion Processes of Charring and NonCharring Solid Fuels, Progress in Energy and Combustion Science 19: 71-104, http://dx.doi.org/10.1016/0360-1285(93)90022-7

[37] Rath, J., Wolfinger, M.G., Steiner, G., Krammer, G., Barontini, F., and Cozzani, V., (2003) Heat of Wood Pyrolysis, Fuel 82: 81-91, http://dx.doi.org/10.1016/S0016-2361(02)00138-2

[38] White, R.H. and Dietenberger, M.A., "Fire Safety of Wood Construction," Chapter 18 in Wood Handbook - Wood as an Engineering Material, Forest Products Laboratory General Technical Report FPL-GTR-190, 2010. 